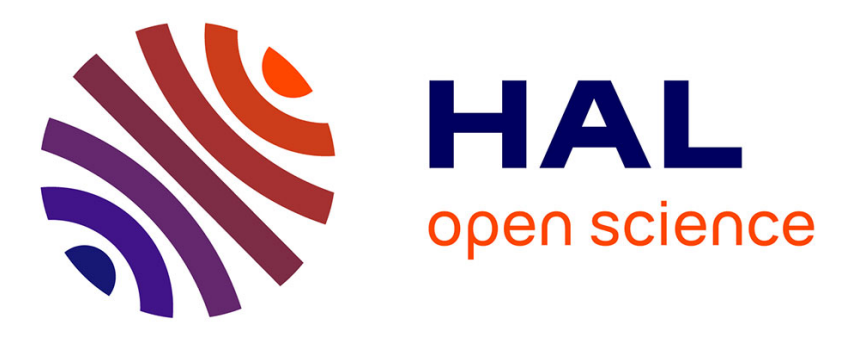

\title{
Dendritic needle network modeling of the Columnar-to-Equiaxed transition. Part I: two dimensional formulation and comparison with theory
}

Pierre-Antoine Geslin, Chih-Hung Chen, Amirhossein Molavi Tabrizi, Alain Karma

\section{To cite this version:}

Pierre-Antoine Geslin, Chih-Hung Chen, Amirhossein Molavi Tabrizi, Alain Karma. Dendritic needle network modeling of the Columnar-to-Equiaxed transition. Part I: two dimensional formulation and comparison with theory. Acta Materialia, 2021, 202, pp.42-54. 10.1016/j.actamat.2020.10.009 . hal03066630

\section{HAL Id: hal-03066630 \\ https://hal.science/hal-03066630}

Submitted on 15 Dec 2020

HAL is a multi-disciplinary open access archive for the deposit and dissemination of scientific research documents, whether they are published or not. The documents may come from teaching and research institutions in France or abroad, or from public or private research centers.
L'archive ouverte pluridisciplinaire HAL, est destinée au dépôt et à la diffusion de documents scientifiques de niveau recherche, publiés ou non, émanant des établissements d'enseignement et de recherche français ou étrangers, des laboratoires publics ou privés. 


\title{
Dendritic Needle Network Modeling of the Columnar-to-Equiaxed Transition. Part I: Two Dimensional Formulation and Comparison with Theory
}

\author{
Pierre-Antoine Geslin ${ }^{\mathrm{a}, *}$, Chih-Hung Chen ${ }^{\mathrm{b}}$, Amirhossein Molavi Tabrizi ${ }^{\mathrm{c}}$, Alain Karma ${ }^{\mathrm{c}, *}$ \\ ${ }^{a}$ Univ. Lyon, INSA-Lyon, MATEIS UMR 5510 CNRS, 69632 Villeurbanne, France \\ ${ }^{b}$ Institute of Applied Mechanics, National Taiwan University, Taipei 106, Taiwan \\ ${ }^{c}$ Department of Physics and Center for Interdisciplinary Research on Complex Systems, Northeastern University, Boston, MA 02115, USA
}

\begin{abstract}
The columnar-to-equiaxed transition is a technologically important phenomenon that controls the grain structure in the casting of alloys. Analytical and coarse-grained models, used to investigate this transition, rely on several assumptions concerning dendrite growth kinetics and grain structures. In the first part of this two part paper, we test these assumptions using a two-dimensional (2D) dendritic needle network model that describes both the transient growth dynamics of primary, secondary and higher order branches of the dendritic network within each grain and the solutal interactions between grains that can grow with arbitrary shapes. Our results provide novel insights into the columnar-to-equiaxed transition, distinguishing between abrupt and progressive transitions with different grain structures. Furthermore, they highlight the limitations of commonly used assumptions in analytical and coarse-grained numerical models of this transition. These results are extended to 3D in part II of the paper.
\end{abstract}

\section{Introduction}

The Columnar-to-Equiaxed transition (CET) is a morphological transition occurring during the solidification of metallic alloys. During the casting of large samples, the development of columnar grains is first favored by the large thermal gradient at the edge of the crucible. This columnar front progresses towards the inside of the sample where lower gradients and slower solidification rates favor the nucleation of equiaxed grains ahead of the columnar front leading to the CET [1].

Because of its technological relevance, this transition has been extensively studied during the past decades. Hunt and Flood [1, 2, 3, 4] proposed the first analytical model to predict the occurrence of the CET. This approach was then extended by Gäumann, Trivedi and Kurz [5] by using more accurate growth laws and by accounting for interactions between equiaxed and columnar grains. In these models, the complex thermal conditions adjacent to the wall of the crucible are simplified by considering that the sample is pulled at a velocity $V_{p}$ in a constant thermal gradient $G$ as in a Bridgman set-up (see Fig. 1

\footnotetext{
${ }^{*}$ Corresponding authors

Email addresses: pierre-antoine.geslin@insa-lyon.fr (Pierre-Antoine Geslin), a. karma@northeastern.edu (Alain Karma)
}

where we denote by $x$ the coordinate in the frame of the sample moving at velocity $V_{p}$ and $x^{\prime}=x-V_{p} t$ the coordinate in a moving frame with fixed isotherms). Both approaches consider the simple case of a dilute binary alloy presenting a linear phase-diagram (see Fig. 1). Analytical models of the CET traditionally rely on several assumptions, which are detailed in Appendix $\mathrm{C}$ for the model of Gäumann et al. [5] and briefly summarized here. Firstly, the columnar front is assumed to reach a steady-state growth regime and stabilizes at a position $x_{c}^{\prime}$ (see Fig. 1) predicted by the combination of the Ivantsov equation and a tip selection criteria [1]. This analytical solution is referred to as the Ivantsov solution in the following. Secondly, the nucleation of equiaxed grains is assumed to occur on foreign particles at a characteristic undercooling, i.e. at a specific position denoted $x_{n}^{\prime}$ in the frame of the isotherms. The equiaxed grains then grow during a time $\left(x_{n}^{\prime}-x_{c}^{\prime}\right) / V_{p}$ before reaching the columnar front. Thirdly, the equiaxed grains are assumed to develop with primary dendrite arms of equal length growing under steady-state conditions with a velocity determined by the ever-changing supersaturation. Lastly, the CET is assumed to occur if the equiaxed grains cover a given fraction $\eta$ of the columnar front. In practice, $\eta$ is chosen between $1 \%$ and $99 \%$, which makes the prediction of the CET imprecise. 

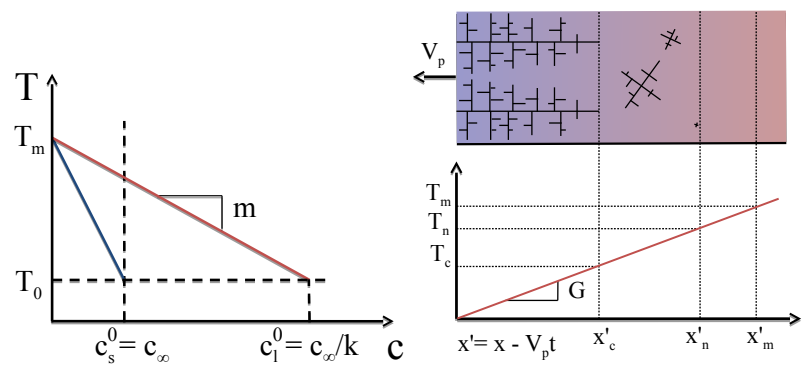

Figure 1: Left: linear phase diagram for a binary alloy displaying the solidus line in blue and the liquidus line in red. Right: diagram depicting the frozen temperature approximation assumed in this study and a typical solidification microstructure forming in the thermal gradient (right plot). The positions $x_{c}^{\prime}, x_{n}^{\prime}$ and $x_{m}^{\prime}$ associated to the frame of the isotherms correspond respectively to the position of the columnar front (at temperature $T_{c}$ ), of the nucleation events (at temperature $T_{n}$ ) and of the melting temperature isotherm (temperature $T_{m}$ ).

Despite incorporating the important physical ingredients, these approaches rely on some strong assumptions, specifically concerning the growth laws of both equiaxed and columnar grains, along with the rather crude criteria to predict the transition. In the past decades, the development of numerical methods in the field of solidification allowed to investigate the CET in more details by relaxing some of these assumptions. Meso-scale models such as volume-averaged based models $[6,7,8]$ and the Cellular Automaton Finite Element method (CAFE) $[9,10]$ allow to investigate the CET in a complex thermal environment, accounting for recalescence and thermal diffusion. However, in these models, the complex dendritic structure of the grains is not resolved and the grains are assumed to follow a steady-state growth; therefore these approaches neglect the influence of the transient regime. Dong and Lee used a Cellular Automaton coupled with Finite Differences (CAFD) to study the CET [11]. This simple model reproduces qualitatively dendritic structures and the main features of the CET. However, it has not been shown to reproduce quantitatively the dendritic transient and steady-state growth regimes. Some authors used the phase-field method to investigate the CET $[12,13]$. This simulation technique accounts for the detailed evolution of the solid-liquid interface and the complexity of solutal interactions but remains limited in terms of length and time scales $[14,15]$, making the study of a meso-scale phenomenon such as the CET numerically challenging.

The dendritic needle network (DNN) model has been recently proposed to study the development of dendritic structures during binary alloys solidification $[16,17]$. In contrast with the phase-field approach, this meso-scale model does not account for the detailed evolution of the solid/liquid interface and relies on a representation of the dendritic structure as an array of needles. It rigorously describes both the transient and steady-state growth regimes of those needles and accounts for the diffusive interactions between them. It allows to bridge scales between phasefield approaches and coarse-grained stochastic models by investigating solidification microstructures on a centimeter length-scale while incorporating the branched structure of the grains and the dynamic of those branches.

In this paper, we use the DNN model to investigate the CET in two dimensions and discuss some assumptions that are used in the analytical and numerical approaches described herein before. Our approach is general but we will use the material parameters pertaining to Al-7wt.\%Si alloy that has been studied extensively in the past $[18,19]$ and is the focus of the ongoing Columnar-to-Equiaxed Transition in SOLidification processing (CETSOL) project [20, 21, 22, 23].

We first present the DNN model used in this study and describe the recent improvements that allow to investigate the nucleation and growth of equiaxed grains. We then discuss the structure of the columnar front and its dependence on the thermal gradient and pulling velocity. Next, we investigate the growth of an equiaxed grain in a thermal gradient and discuss how it compares with the steadystate growth regime assumed in the analytical models of CET. Finally, we investigate the occurrence of the CET and compare our results to the analytical prediction of the model of Ref. [5] adapted in 2D.

\section{Dendritic needle network (DNN) model}

We now present the DNN model introduced in [16] and [17]. In this approach, a dendrite is represented by a needle with its dynamics determined by two conditions prescribing its tip velocity $V$ and radius $\rho$. First, we rely on the micro-solvability condition which relates the product $\rho^{2} V$ to the strength of the surface tension anisotropy [24]. Phase-field simulations proved that this product reaches a constant value very rapidly during the transient stage of dendritic growth [25] while $\rho$ and $V$ are still far from their steady state values. The second condition is determined on a larger scale than the tip radius but smaller than the typical diffusion length, where a flux intensity factor (FIF) noted $\mathcal{F}$ can be computed from a contour integral and measures the strength of the solute flux directed towards the tip. As shown in Sec. 2.1, solute conservation at the dendritic tip requires that $\rho V^{2} \sim \mathcal{F}^{2}$. These two relations determine uniquely the tip radius $\rho$ and velocity $V$ for each dendritic branch. The advantage of the DNN model lies in its capacity to capture accurately the 
transient growth regime as well as the solute interactions between dendrites, using a numerical grid spacing of the same order as the tip radius, i.e. orders of magnitude larger than quantitative phase-field simulations.

The first formulation of the DNN model [16] relies on a description of the needles as width-less segments. One of the limitations of this description is that the needles have to be positioned along the main direction of the numerical grid used to compute the evolution of the solute field in order to set properly the boundary conditions for the solute field. Therefore, this model cannot represent grains of different orientations as encountered in the CET. This limitation has been overcome recently with a thick formulation of the model where the needles are represented as parabolas of finite width [17]. This new model has also been shown to reproduce the stationary growth rate over a wider range of solute tip supersaturations. In the following, this formulation of the model is described.

\subsection{Sharp-interface model for directional solidification}

We use $c$ to denote the solute concentration in the liquid and more specifically $c_{l}\left(c_{s}\right)$ for the equilibrium concentration on the liquid side (solid side) of the solid-liquid interface given by the alloy phase-diagram. We consider a binary alloy of nominal composition $c_{\infty}$ in the dilute limit such that the phase diagram is assumed linear (see Fig. 1). In particular, we note $m$ the slope of the liquidus curve, $k=c_{s} / c_{l}$ the partition coefficient, and $T_{0}$ the reference temperature defined where the solidus line reaches the nominal composition $c_{\infty}$.

We are interested in the case of directional solidification where the sample is being pulled at a constant rate $V_{p}$ into a fixed thermal gradient $G$ (see Fig. 1). Because the thermal diffusivity in both liquid and solid phases is order of magnitude larger than the solute diffusivity, we consider the frozen temperature approximation in which the temperature field is given by

$$
T(x, t)=T_{0}+G\left(x-V_{p} t\right)
$$

where $x$ is a coordinate attached to the sample being pulled at a speed $V_{p}$.

The Gibbs-Thomson relation [1] gives the melting temperature of a curved interface as a function of materials parameters:

$$
T=T_{m}+m c_{l}-\Gamma_{s l} f(\theta) \kappa
$$

where $T_{m}$ is the melting temperature of pure aluminum, $f(\theta)$ measures the angular dependence of the interface stiffness and $\Gamma_{s l}$ is the Gibbs-Thomson coefficient [1]. The concentration on the liquid side of a solid/liquid interface is space-dependent and is obtained by combining the Gibbs-Thomson relation with Eq. (1):

$$
\frac{c_{l}}{c_{l}^{0}}=1-(1-k) d_{0} f(\theta) \kappa-(1-k) \frac{x-V_{p} t}{l_{T}}
$$

where $c_{l}^{0}=c_{\infty} / k, d_{0}=\Gamma_{s l} /\left(|m|(1-k) c_{l}^{0}\right)$ is the capillary length and $l_{T}=|m|(1-k) c_{l}^{0} / G$ is the thermal length related to the strength of the thermal gradient.

The solute concentration in the liquid is assumed to follow a simple diffusion equation with diffusivity $D$ :

$$
\partial_{t} c=D \nabla^{2} c
$$

Neglecting diffusion in the solid phase, solute conservation is expressed by the usual Stefan condition

$$
(1-k) c_{l} v_{n}=-\left.D \partial_{n} c\right|_{i}
$$

where $v_{n}$ denotes the interface normal velocity, $\left.\partial_{n} c\right|_{i}$ is the normal solute gradient taken at the interface and $c_{l}$ is given by Eq. (3). Equations (3-5) define the free-boundary problem associated with the evolution of the solid/liquid interface in the presence of a temperature gradient.

To reduce the number of parameters involved in this free-boundary problem, we introduce the reduced solute concentration field defined as

$$
u=\frac{c_{l}^{0}-c}{(1-k) c_{l}^{0}} .
$$

The sharp interface problem then translates into

$$
\begin{aligned}
& \partial_{t} u=D \nabla^{2} u \\
& \left(1-(1-k) u_{i}\right) v_{n}=\left.D \partial_{n} u\right|_{i}
\end{aligned}
$$

where the field $u$ is subjected to a boundary condition along the solid/liquid interface given by the GibbsThomson relation:

$$
u_{i}(x, t)=d_{0} f(\theta) \kappa+\frac{x-V_{p} t}{l_{T}} .
$$

In the following, we will consider that, on the scale of the needles, far from their tip, the effect of the curvature is negligible and we approximate Eq. (9) by:

$$
u_{i}(x, t)=\frac{x-V_{p} t}{l_{T}} .
$$

An additional boundary condition is simply obtained by considering the composition of the alloy far from the solid:

$$
u(x \rightarrow+\infty, t)=1
$$


As explained in earlier descriptions of the model [16, 17], the free-boundary problem defined herein before admits an unique solution around a parabolic tip only if the product $\rho^{2} V$ is fixed to a constant:

$$
\rho^{2} V=\frac{1}{1-(1-k) u_{t}} \frac{2 D d_{0}}{\sigma}
$$

where $\sigma$ is a tip selection parameter uniquely defined by the strength of the crystalline anisotropy and $u_{t}$ is the concentration at the solid/liquid interface at the tip of the needle given by Eq. (10).

A second condition relates the product $\rho V^{2}$ to the strength of the solute flux directed towards the dendritic tip. To derive it, we consider a needle of tip radius $\rho$ growing at a velocity $V$ toward a direction representing with a unit vector $\boldsymbol{x}_{n}$ as depicted in Fig. 2. In the moving frame associated to the needle, the Stefan condition along the needle interface is written as

$$
V \boldsymbol{x}_{n} \cdot \boldsymbol{n}_{n}=\frac{D}{1-(1-k) u_{t}} \partial_{n} u
$$

We integrate this equation on the needle interface along the contour $\Gamma_{n}$ following the parabolic tip up to distances $a_{1}$ and $a_{2}$ on both sides of the needle (see Fig. 2). We obtain:

$$
V \sqrt{2 \rho}\left(\sqrt{a_{1}}+\sqrt{a_{2}}\right)=\frac{D}{1-(1-k) u_{t}} \int_{\Gamma_{n}} \partial_{n} u \mathrm{~d} \Gamma
$$

which can be rewritten as

$$
\rho V^{2}=\frac{2 D^{2} \mathcal{F}^{2}}{\left[1-(1-k) u_{t}\right]^{2} d_{0}}
$$

where $\mathcal{F}$ is the flux intensity factor defined as:

$$
\mathcal{F}=\frac{\sqrt{d_{0}}}{2\left(\sqrt{a_{1}}+\sqrt{a_{2}}\right)}\left(\int_{\Gamma_{n}} \partial_{n} u \mathrm{~d} \Gamma\right) .
$$

As detailed in Appendix A, expressions for the lengths $a_{1}$ and $a_{2}$ can be deduced from elementary geometry.

The boundary integral appearing in this equation can be computed by considering the integration contour $\Gamma_{b}$ depicted in blue in Fig. 2. On the scale of the contour $\Gamma$, we can consider that the diffusion equation reduces to its Laplacian limit. In the moving frame associated to the needle, this yields:

$$
D \nabla^{2} u+\boldsymbol{V} \cdot \boldsymbol{\nabla} u=0
$$

that we integrate on the area $\Sigma$ delimited by the closed contour $\Gamma=\Gamma_{n}+\Gamma_{b}$. Applying the divergence theorem yields ${ }^{1}$ :

$$
\int_{\Gamma_{b}} \boldsymbol{\nabla} u \cdot \boldsymbol{n}_{b} \mathrm{~d} \Gamma-\int_{\Gamma_{n}} \partial_{n} u \mathrm{~d} \Gamma=-\frac{1}{D} \iint_{\Sigma} \boldsymbol{V} \cdot \boldsymbol{\nabla} u \mathrm{~d} \Sigma
$$

This equation relates the integral on the parabolic contour $\Gamma_{n}$ to an integral on a regular contour $\Gamma_{b}$ and a surface integral. In practice, the contour $\Gamma_{b}$ is chosen to be aligned with the numerical finite difference grid used to discretize the field $u$. Therefore, the integrands of Eq. (18) can be easily estimated from finite differences as detailed in Refs. [16, 17].

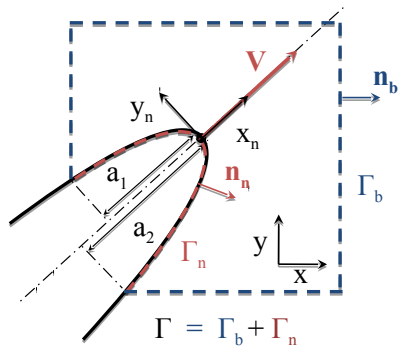

Figure 2: Schematics presenting a parabolic needle tip and the contours used to compute the flux intensity factor. The needle is growing at velocity $V$ toward a direction with unit vector $\boldsymbol{x}_{n} ; \boldsymbol{n}_{n}$ is the unit normal everywhere on the needle. $\Gamma_{n}$ is the needle boundary and $\Gamma_{b}$ is a regular rectangular boundary around the tip. The surface bounded between $\Gamma_{b}$ and $\Gamma_{n}$ is denoted $\Sigma$. The boundary $\Gamma_{b}$ meet the parabolic tip up to distances $a_{1}$ and $a_{2}$.

As in previous implementations of the model $[16,17]$, side-branching is assumed to occur at a distance $d \sim N \times \rho$ behind the tip of the needle every time the tip has grown a length $d$. Experiments show that in 2D, $N$ is of the order of 5-10 [26]. To reproduce the stochastic aspects of the sidebranching process observed experimentally, the distance $d$ is picked randomly in $(N \pm \Delta N / 2) \rho$. When a new branch is created, its length is set to an arbitrary small length of $12 \rho_{s}$.

\subsection{Steady-state solution}

To find the steady-state tip radius $\rho_{s}$ and its position in the thermal gradient, we examine the free-boundary problem described previously in the vicinity of a parabolic tip. We consider an intermediate length-scale large compared to the tip radius and small compared to the thermal

\footnotetext{
${ }^{1}$ We point out here the change of sign associated to the different definition of the normals on the contour $\Gamma_{b}$ (outer-pointing normal) and $\Gamma_{n}$ (inward-pointing normal)
} 
length. On this scale, the concentration at the solid/liquid interface can be considered as constant and equal to $u_{t}=$ $\left(x_{t}-V_{p} t\right) / l_{T}$, where $x_{t}$ is the position of the tip. Within this assumption, the free-boundary problem admits a single solution given by the Ivantsov relation between the Péclet number $P e=\rho_{s} V_{p} / 2 D$ and the tip supersaturation $\Omega_{s}=\left(1-u_{s}\right) /\left(1-(1-k) u_{s}\right)$. Thus, the stationary state can be determined by solving the following set of non-linear equations for $\Omega_{s}, u_{s}$ and $\rho_{s}$ :

$$
\begin{aligned}
\rho_{s} & =\sqrt{\frac{1}{1-(1-k) u_{s}} \frac{2 D d_{0}}{\sigma V_{p}}} \\
\Omega_{s} & =\operatorname{Iv}\left(\frac{\rho_{s} V_{p}}{2 D}\right) \\
u_{s} & =\frac{1-\Omega_{s}}{1-(1-k) \Omega_{s}}
\end{aligned}
$$

where $I v$ denotes the Ivantsov function in 2D: $I v(P e)=$ $\sqrt{\pi P e} \exp (P e) \operatorname{erfc}(\sqrt{P e})$. The steady-state values $\Omega_{s}, u_{s}$, and $\rho_{s}$ are found by solving numerically this set of equation with a fixed-point iterative method. The steady-state tip undercooling $\Delta T_{S}$ is a more widely used measure of the needle's position in the thermal gradient. It is related to $u_{s}$ by the simple relation:

$$
\Delta T_{s}=\left(1-u_{s}\right) G l_{T}
$$

\subsection{Dimension-less equations and numerical implemen- tation}

The characteristic time-scale and length-scales are defined as $l_{c}=\rho_{s}$ and $t_{c}=\rho_{s} / V_{p}$ and the dimension-less values are denoted with a tilde $(\sim)$. The diffusion equation becomes

$$
\partial_{\tilde{t}} u=\tilde{\nabla}^{2} u
$$

The equations for the needle evolution reduce to:

$$
\begin{aligned}
& \tilde{\rho}^{2} \tilde{V}=\frac{1-(1-k) u_{s}}{1-(1-k) u_{t}} \\
& \tilde{\rho} \tilde{V}^{2}=\frac{2 \tilde{D}^{2}}{\left(1-(1-k) u_{t}\right)^{2}} \tilde{\mathcal{F}}^{2}
\end{aligned}
$$

where the dimension-less flux intensity factor $\tilde{\mathcal{F}}$ is defined $\mathrm{as}^{2}$ :

\footnotetext{
${ }^{2}$ We point out here the difference between the concentration at the tip $u_{t}$ that may vary depending on the position of the needle in the thermal gradient and its steady-state value $u_{s}$ given by Eq. (21).
}

$$
\tilde{\mathcal{F}}=\frac{1}{2\left(\sqrt{\tilde{a_{1}}}+\sqrt{\tilde{a_{2}}}\right)}\left[\int_{\Gamma_{b}} \tilde{\nabla} u \cdot \boldsymbol{n}_{b} \mathrm{~d} \Gamma+\frac{1}{\tilde{D}} \iint_{\Sigma} \tilde{\boldsymbol{V}} \cdot \tilde{\nabla} u \mathrm{~d} \Sigma\right]
$$

Equations (24-25) allow to compute the velocity and tip radius of the needle at each time-step.

The solute field is discretized on a finite difference square grid of spacing $\Delta x$ and the diffusion equation Eq. (23) is solved using finite differences and a forward Euler scheme.

An integer field $\phi$ is also introduced to keep track of the area occupied by the needles on the finite difference grid ( $\phi=1$ in the liquid and $\phi=0$ on the needles). During the evolution of the microstructure, $\phi$ is updated according to the new position of each needle tip and its radius. We consider that the solid/liquid interface adopts a parabolic shape only close to the tip; therefore, $\phi$ is set to 0 on the area included in a truncated parabola of radius $\rho$ and length $L_{t r}$. To reduce computational costs, we note that the field $\phi$ is not updated according to the lateral fluxes on the sides of the needle and does not keep track of the evolution of the solid/liquid interface far from the tip. This simplification has been shown to have a small influence on the steady-state growth of the needle [17]. The introduction of the field $\phi$ also makes easier the implementation of the boundary condition of Eq. (10) on the grid points occupied by the needles. The evolution of the solute field and the needles are integrated using a GPU implementation of the model. In the following, we will consider material parameters pertaining to $\mathrm{Al}-7 \mathrm{wt}$.\% Si as listed in table 1. To investigate the CET in different conditions, we will consider thermal gradients between $G=1 \mathrm{~K} / \mathrm{mm}$ and $10 \mathrm{~K} / \mathrm{mm}$ and puling velocities between $V_{p}=10 \mu \mathrm{m} / \mathrm{s}$ and $100 \mu \mathrm{m} / \mathrm{s}$.

In the following, we take $\Delta x=\rho_{s}$ leading to an accurate representation of the needles on the finite difference grid. We point out the multi-scale character of the DNN model that is able to investigate microstructures evolution influenced by four different length-scales listed in Tab. 1 spanning from the nanoscale capillary length $d_{0}$ up to the thermal length of the order of centimeter.

\subsection{Reproduction of the steady-state growth}

To check that the implementation of the model reproduces correctly the expected steady-state growth for various needle orientations, we introduce a single needle in a gradient $G=1 \mathrm{~K} / \mathrm{mm}$ oriented along the $x$-axis such that the growth direction of the needle makes an angle $\theta$ with $x$. We use the parameters of Tab. 1 and prohibit sidebranching. Figure 3 displays the resulting steady-state tip supersaturation $\Omega_{s}$ function of the Péclet number defined 


\begin{tabular}{lll}
\hline Symbol & Value & Unit \\
\hline$c_{\infty}$ & 7 & $\mathrm{wt} . \% \mathrm{Si}$ \\
$m$ & -6.5 & $\mathrm{~K} \mathrm{wt} \% \mathrm{Si}$ \\
$k$ & 0.13 & \\
$\Gamma_{s l}$ & $1.96 \cdot 10^{-7}$ & $\mathrm{~K} \mathrm{~m}$ \\
$D$ & $3 \cdot 10^{-9}$ & $\mathrm{~m}^{2} / \mathrm{s}$ \\
$\sigma$ & 0.83 & \\
$G$ & $1-10$ & $\mathrm{~K} / \mathrm{mm}$ \\
$V_{p}$ & $10-100$ & $\mu \mathrm{m} / \mathrm{s}$ \\
\hline$d_{0}$ & $6.44 \cdot 10^{-10}$ & $\mathrm{~m}$ \\
$\rho_{s}$ & $1.68 \cdot 10^{-6}-5.57 \cdot 10^{-6}$ & $\mathrm{~m}$ \\
$l_{D}$ & $3.00 \cdot 10^{-5}-3.00 \cdot 10^{-4}$ & $\mathrm{~m}$ \\
$l_{T}$ & $3.05 \cdot 10^{-2}-3.05 \cdot 10^{-1}$ & $\mathrm{~m}$ \\
\hline$N$ & 15 & \\
$\Delta N$ & 10 & \\
$\Delta x / \rho_{s}$ & 1 & \\
$L_{t r} / \rho_{s}$ & 12 & \\
\hline
\end{tabular}

Table 1: Parameters used in the following to model directional solidification of Al-7wt.\%Si $[18,19]$ for various thermal gradient and pulling velocities.

as $P e=\rho_{s} V_{p} / 2 D$ and compared to the Ivantsov relation. For the various orientations, the model reproduces rather accurately the steady-state growth regime predicted by the Ivantsov solution for intermediate Péclet numbers. For $P e \lesssim 0.008$, the dependence of $u_{i}$ on $x$ presented in Eq. (9) becomes non-negligible on the scale of the tip and affects the concentrations profiles and the tip supersaturation. The numerical results also differ from the Ivantsov solution for high values of the Péclet number. In this limit, the diffusion equation deviates from its Laplacian approximation in the vicinity of the tip where the flux intensity factor is computed.

\section{Nucleation and growth from inoculants}

As shown previously, the DNN model accurately accounts for the growth of dendritic arms of well developed grains but the nucleation and the first stage of globular growth of new grains is an important ingredient of the CET that has to be incorporated in the model.

\subsection{Nucleation}

In industrial casting, foreign particles are deliberately added to the melt (e.g. $\mathrm{TiB}_{2}$ particles in $\mathrm{Al}-\mathrm{Si}$ alloys) to promote heterogeneous nucleation and the development of a refined microstructure made of small grains [1]. As described in the model of Greer et al. [27, 28], these inoculant particles are chosen for their ability to host a cap of solid. When this cap grows on the particle surface, its

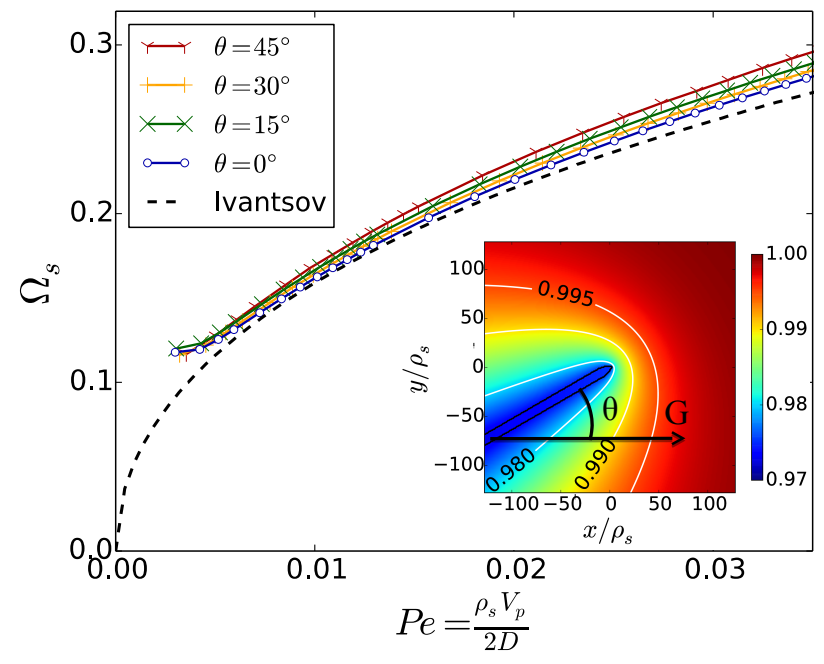

Figure 3: Stationary values of the Péclet number $P e$ and the tip supersaturation $\Omega_{s}$ obtained numerically for different orientation $\theta$ between the needle and the thermal gradient. The inset depicts iso-concentration contours around a needle.

curvature reaches a maximum for a hemispherical shape of the same radius than the particle. Above this critical shape, a free growth condition is reached and a grain develops. Therefore, in this framework, the nucleation undercooling of these particles is inversely proportional to their radius $R_{i}: \Delta T_{n}=2 \Gamma_{s l} / R_{i}$ [1]. Considering inoculant particles of radius $R=2 \mu \mathrm{m}$ [1] lead to a characteristic undercooling $\Delta T_{n}^{(1)}=0.196 \mathrm{~K}$. In practice, experiments have shown that, for the case of $\mathrm{TiB}_{2}$ particles in Al-Si alloys, poisoning of the inoculant particles in presence of $\mathrm{Si}$ increases considerably their undercooling up to $\Delta T_{n}^{(2)}=8 \mathrm{~K}[29,30]$.

In the following, we will consider a homogeneous density $c_{\text {inoc }}$ (ranging from $c_{\text {inoc }}=0.125 \mathrm{~mm}^{-2}$ to $c_{\text {inoc }}=$ $1.25 \mathrm{~mm}^{-2}$ ) of inoculant particles presenting a unique undercooling (either $\Delta T_{n}^{(1)}$ or $\Delta T_{n}^{(2)}$ ) with no statistical distribution. However, incorporating a realistic distribution (e.g. log-normal) of undercooling on the inoculant population would be straightforward.

The growth of a new grain on a inoculant particle is initiated when its nucleation undercooling $\Delta T_{n}$ becomes smaller than the local undercooling at its position $\boldsymbol{r}$ defined as the difference between composition-dependent liquidus temperature and the actual temperature at the position in the thermal gradient:

$$
\Delta T_{n}<\left(T_{m}-|m| c(\boldsymbol{r})\right)-\left(T_{0}+G\left(x-V_{p} t\right)\right) .
$$

Dividing this equation by $|m|(1-k) c_{l}^{0}$ makes appear the reduced solute field $u(\boldsymbol{r})$ and its value on the solid/liquid 
interface $u_{i}(x, t)$ defined by Eq. (10). After reorganization of the terms, we obtain:

$$
u(\boldsymbol{r})>u_{i}(x, t)+\frac{\Delta T_{n}}{|m|(1-k) c_{l}^{0}} .
$$

When this condition is fulfilled, nucleation occurs and the inoculant particle is replaced by a circular grain.

\subsection{Circular growth}

Before presenting well-developed dendritic arms whose evolution can be treated within the DNN formalism, the newly nucleated particles are modeled as growing circular grains. The influence of the solutal environment on the growth rate of these grains can be taken into account in a similar way as for dendritic growth: the incoming solute flux on the particle is computed with the use of a contour integral around the grain (see Fig. 4.a). The growth rate of the grain is given by the Stefan condition

$$
v_{g}=D \partial_{n} u
$$

where $n$ denotes the normal to the grain surface pointing outward. Integrating this relation on the closed contour $\Gamma_{g}$ represented in Fig. 4.a yields:

$$
2 \pi R_{g} v_{g}=-D \int_{\Gamma_{g}} \partial_{n} u \mathrm{~d} \Gamma_{g} .
$$

On the scale of the contour $\Gamma$ close to the solid/liquid interface, the diffusion equation can be approximated by its Laplacian limit $\nabla^{2} u=0$. Integrating the Laplace equation on the surface delimited by the contour $\Gamma$ and applying the divergence theorem yields:

$$
\int_{\Gamma} \boldsymbol{\nabla} u \cdot \boldsymbol{n} \mathrm{d} \Gamma=0
$$

Considering that $\Gamma=\Gamma_{g}+\Gamma_{e}$, we then obtain the following relation between the growth velocity $v$ and the incoming flux integrated along $\Gamma_{e}$ :

$$
v=\frac{D}{2 \pi R} \int_{\Gamma_{e}} \nabla u \cdot \boldsymbol{n}_{e} \mathrm{~d} \Gamma_{e}
$$

Similar to the case of needles, this integral is easily computed numerically on the finite difference grid. The time evolution of the circular grain radius is then deduced from the numerical integration of this equation with a forward Euler scheme. This method enables to take naturally into account the interactions of the growing grains with their solutal environment as well as their transient growth regime.

In isothermal conditions corresponding to the limit $G \rightarrow 0$, the stationary growth regime can be compared to the Zener solution [31] of a growing 2D circle given by the relation $\Omega=p e^{p} \int_{p}^{+\infty} e^{-x} / x d x$ where $p=R_{g} v_{g} / 2 D$ is the Péclet number associated to the germ growth and $\Omega=\left(c_{l}^{0}-c_{\infty}\right) /\left((1-k) c_{l}^{0}\right)$ is the supersaturation. Figure 4.b shows that the numerical results follow the Zener solution for small Péclet numbers and deviates slightly for higher Péclet numbers where the Laplacian approximation of the diffusion equation becomes less accurate.

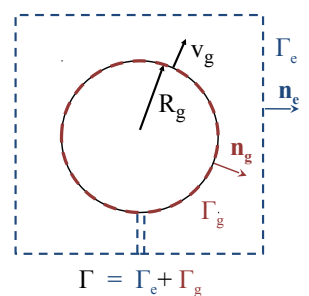

(a)

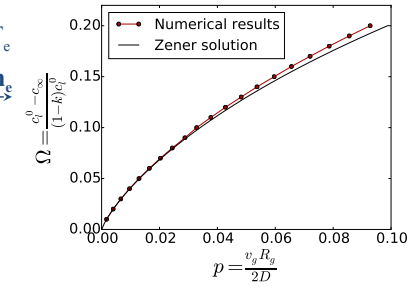

(b)
Figure 4: (a) Computation of the growth rate of the circular grain from the integration of the incoming solutal flux. (b) Comparison between numerical results and analytical solution of Zener [31] for the growth of a circular grain in a supersaturated environment.

Analytical approaches and phase field models [1, 25] show that, for a critical radius (that depends on the solutal environment), the solid/liquid interface of a small spherical grain destabilizes, leading to the development of small dendritic arms. However, the DNN model can only follow the growth of well-developed dendrites and is not suited to investigate the first stages of destabilization. Therefore, for simplicity reasons, we suppose that the destabilization occurs for a large critical radius $R_{c}=8.7 \rho_{s}\left(\rho_{s}\right.$ is the stationary state tip radius), regardless of the solutal environment. When the circular grain radius reaches this threshold, it is replaced by an equiaxed grain made of four needles of tip radius $\rho_{s}$ and presenting an angle $\pi / 2$ between each other. The length of the dendritic arms is chosen such that the solid fraction of the grain is conserved between its circular and needle representations. The value of the threshold $R_{c}=8.7 \rho_{s}$ is chosen because the resulting length of the needles $12 \rho_{s}$ is large enough to be treated accurately with the DNN approach. To justify our simplification of a fixed value of $R_{c}$, we show in Appendix B that $R_{c}$ affects the early stages of grain growth but has a marginal influence on the consecutive development of the grain.

\section{Columnar front}

In analytical models for CET and most coarse-grained numerical models, the position of the columnar front in the thermal gradient is generally estimated by considering that the front is composed of non-interaction parabolic 
tips whose steady-state dynamics satisfies the Ivantsov relation. However, the presence of side-branches and neighboring dendrites influence the solute field around the primary dendrites and therefore their stationary states. In this section, we investigate the steady-state regime of the columnar front with the DNN model and discuss these effects.

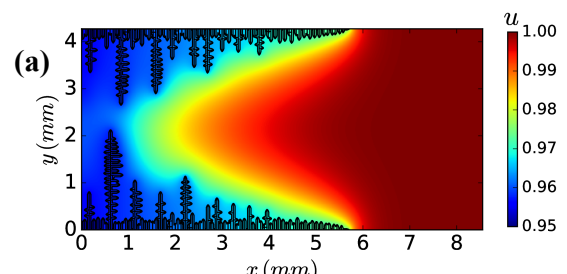

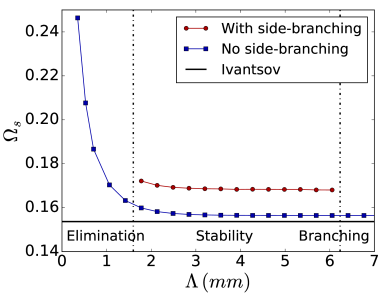

(b)

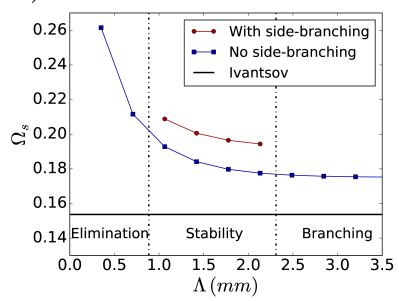

(c)
Figure 5: (a) Dendritic structure obtained for a primary spacing $\Lambda=$ $4.28 \mathrm{~mm}$ with $V_{p}=10 \mu \mathrm{m} / \mathrm{s}$ and $G=1 \mathrm{~K} / \mathrm{mm}$; the color map shows the concentration field $u$. (b,c) Primary dendrite tip supersaturation $\Omega_{s}$ function of the primary spacing $\Lambda$ when side-branching is inhibited (blue) and activated (red) for $G=1 \mathrm{~K} / \mathrm{mm}$ (b) and $G=10 \mathrm{~K} / \mathrm{mm}$ (c). The Ivantsov solution is the horizontal black line in (b) and (c).

We consider first the simulation set-up depicted in Fig. 5.a where two needles are introduced on the bottom and top side of the domain. We impose mirror boundary conditions along the $y$-axis such that the primary spacing $\Lambda$ is controlled through the height of the simulation domain. For simplicity, we investigate only one pulling velocity $V_{p}=10 \mu \mathrm{m} / \mathrm{s}$. We first consider a gradient $G=1 \mathrm{~K} / \mathrm{mm}$ and forbid the apparition of secondary branches. The tip supersaturation $\Omega_{s}$ of the primary dendrites $^{3}$ is then reported in Fig. 5.b (square blue data points) as a function of the primary spacing $\Lambda$ and compared to Ivantsov relation (horizontal black line). For low primary spacings (typically $\Lambda<2000 \mu \mathrm{m}$ ), the neighboring dendrites influence significantly the solute field, leading to an increase of the tip supersaturation and a significant deviation from the Ivantsov relation. However, for higher spacings, the influence of the neighboring dendrites be-

\footnotetext{
${ }^{3}$ We note that the columnar front undercooling $\Delta T_{c}$ is directly related to $\Omega_{s}$ through an increasing non-linear function $\Delta T_{c}\left(\Omega_{s}\right)=$ $\left(G k \Omega_{s} l_{T}\right) /\left(\left(1-(1-k) \Omega_{s}\right)\right)$ deduced by combining Eq. (22) and (21)
}

comes negligible and the Ivantsov solution predicts accurately the tip supersaturation.

Then, we test the influence of side-branching on the tip undercooling. In this case, only a range of spacings is stable: for low spacings, the secondary branches influence the solute field and break the symmetry of the simulation domain, leading to the elimination of one of them. When the spacing between primary dendrites is large enough, a new leading branch can appear through the development of a tertiary branch that escapes the inter-dendritic zone, then reducing the primary spacing by half. As shown in Fig. 5.b, the presence of higher-order branches influences significantly the steady-state supersaturation that is about $10 \%$ higher than the Ivantsov solution. Figure 5.c depicts the results obtained for a larger thermal gradient $G=10 \mathrm{~K} / \mathrm{mm}$. The effects presented previously are intensified: in the presence of secondary-branching, the Ivantsov solution underestimates the tip supersaturation by more than $20 \%$.

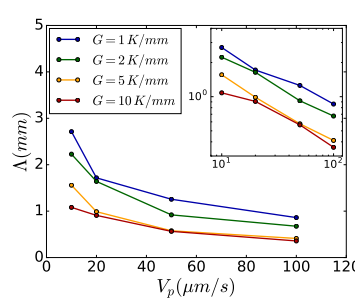

(a)

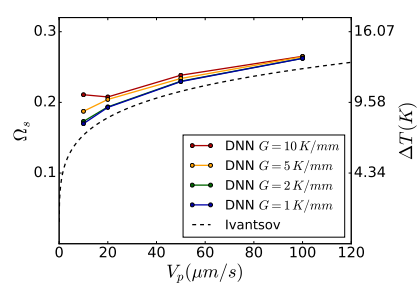

(b)
Figure 6: (a) Primary spacing naturally selected from the destabilization of a planar front for different values of the thermal gradient $G$ and pulling velocity $V_{p}$. The inset shows the same plot in a log-log scale. (b) Supersaturation of the columnar front function of $V_{p}$ for different values of $G$ compared with the Ivantsov solution shown with a dashed line.

Next, we investigate the properties of the columnar front when it develops naturally after the destabilization of a planar interface (modeled as a tight array of needles in our simulations). Solutal interactions between neighboring needles lead to dendrite eliminations and an interdendritic spacing is naturally selected. Figure 6.a shows the average primary spacing as a function of the pulling velocity for different values of the thermal gradient. As expected, the inset of Fig. 6.a shows that the primary spacing decreases with $V_{p}$ following a power-law with an exponent between $-1 / 2$ and $-1 / 4$ [1]. Figure $6 . b$ shows the columnar front supersaturation (or equivalently its undercooling shown in the right $\mathrm{y}$-axis) as a function of $V_{p}$ for different $G$ values, compared to the Ivantsov solution of an isolated dendrite in 2D. Similar conclusions to the previous ones can be drawn: the analytical solution underestimates the columnar front undercooling, specifically for low pulling velocity and large gradient. For high veloc- 
ities, the influence of the thermal gradient vanishes because the scale of the tip radius (and also of the dendritic microstructure) decreases and becomes small compared to the thermal length.

\section{Equiaxed growth in a temperature gradient}

In analytical models for CET, the growth of the equiaxed grains is assumed to follow the Ivantsov relation in a time-dependent temperature environment. In other words, the duration of the transient growth is considered to be negligible compared to the rate of change of temperature $\partial_{t} T=G V_{p}$ and the equiaxed grains to reach a steady-state regime instantly on the time-scale of the temperature evolution. To derive the growth rate of the grains predicted within this assumption, we consider a grain originally placed at the liquidus isotherm. We focus on the vertical branches of the grain that are assumed to grow perpendicularly to the thermal gradient. The interactions between the different branches of the grain are neglected and the growth rate is considered to follow the Ivantsov solution of a parabolic tip growing in a super-saturated liquid. The super-saturation is given by $\Omega(t)=\left\{c_{l}(t)-c_{\infty}\right\} /\left\{(1-k) c_{l}(t)\right\}$, where $c_{l}(t)=$ $\left(T_{m}-T_{0}-G\left(x-V_{p} t\right)\right) /|m|$ is the time-dependent liquidus concentration and the Péclet number is uniquely determined by the Ivantsov relation $\Omega(t)=I v(P e(t))$. The combination of this relation with the micro-solvability condition in isothermal condition $\rho^{2} V=2 D d_{0} / \sigma$ allows to determine the growth velocity of the branches. This velocity, shown with a dashed black line in Fig. 7.a increases sharply with time. The length of the branches (dashed black in Fig. 7.b) is obtained after time integration of the velocity and also shows a sharp increase in time.

To discuss the validity of this behavior, we perform DNN simulations in the most favorable case of a slowly varying temperature environment by choosing a low gradient $G=1 \mathrm{~K} / \mathrm{mm}$ and a low pulling velocity $V_{p}=$ $10 \mu \mathrm{m} / \mathrm{s}$. Initially, a small grain formed of four dendritic arms of initial length $L(t=0)=12 \rho_{s}=66.82 \mu \mathrm{m}$ is introduced at the position of the melting temperature isotherm. We consider three different grain structures:

1. A grain consisting of two vertical needles growing in opposite direction without any side-branching (see Fig. 7.c),

2. A cross shaped grain formed of four perpendicular needles well oriented in the thermal gradient. No side-branching is allowed on any of the branches (see Fig. 7.d).

3. A cross shaped grain oriented as previously but where side-branching is allowed (see Fig. 7.e).

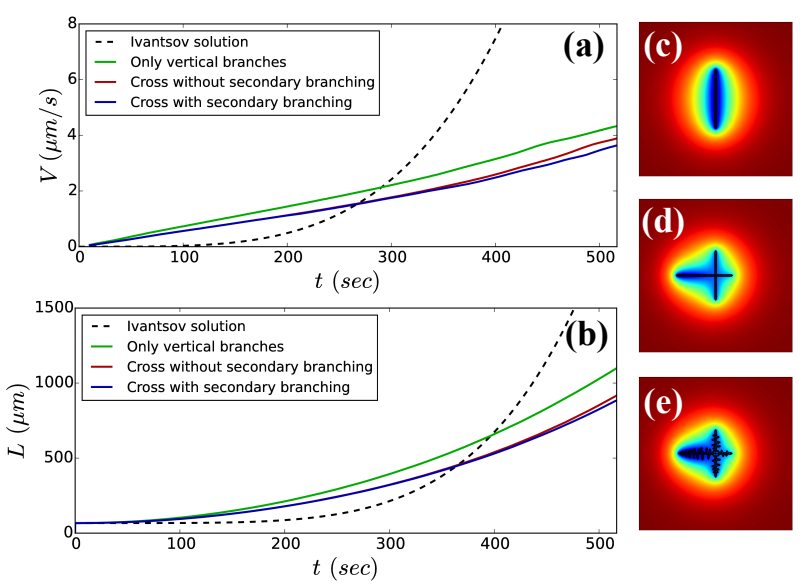

Figure 7: (a,b) Time evolution of the velocity (a) and the length (b) of the vertical branches for $G=1 \mathrm{~K} / \mathrm{mm}$ and $V_{p}=10 \mu \mathrm{m} / \mathrm{s}$ and for different simplified geometries: only vertical branches (green line), cross-shaped grain without side-branching (red) and with side-branching (blue) compared with Ivantsov prediction (dash black line). (c-e) The grain morphology at the end of the simulation and the surrounding solute field are represented for each of these cases.

For these three cases, the velocity and the length of the vertical branches are shown in Figs. 7.a and 7.b and do not follow at all the behavior expected by the analytical solution described above. This can be explained by examining Fig. 8 that displays the concentration profiles along the upper vertical needle of case 1 and the profile predicted by the Ivantsov solution. In our DNN simulations, the branches are first in a transient growth regime and grow faster than predicted by the Ivantsov solution because of the surrounding excess of solute (see Fig. 8.a). With increasing time and supersaturation, the concentration profile predicted by the Ivantsov solution becomes steeper, regardless of the history of the solutal environment. Our model realistically accounts for the time evolution of the profile, showing that it does not adapt instantaneously to the supersaturation. Consequently, at longer time, the concentration profile is much less steep than predicted by the Ivantsov solution leading to a lower velocity as shown in Fig. 7.a.

In summary, in our simulations, the time scale associated with the transient growth regime is far from being negligibly small compared to the time scale on which temperature and supersaturation evolve. Obviously, this is also the case for the higher values of $V_{p}$ and $G$ investigated in this paper. The time evolution of the velocity for the different grain structures depicted in Fig. 7.a shows that the solutal interactions from horizontal branches and secondary branches lower sensibly the velocity of the 


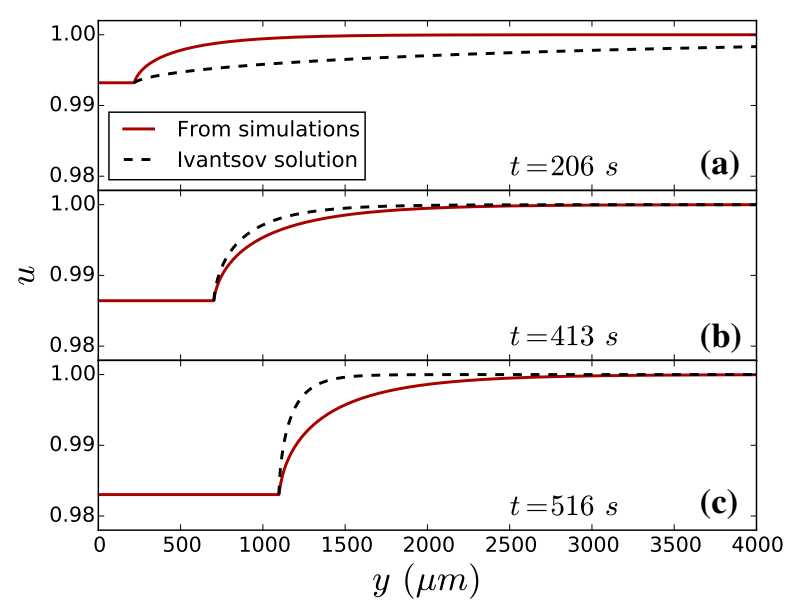

Figure 8: At different times during the growth of the isolated vertical branches (case 1 depicted in Fig. 7), the concentration profile along the $y$-axis is plotted in red and compared to the prediction of the Ivantsov solution in dashed black.

primary vertical branches but does not change the main trends discussed previously. Also, identical conclusions can be drawn for misoriented grains.

The DNN model allows to investigate the more complicated case of interacting equiaxed grains during the CET and the influence of the realistic circular germ regime on the resulting microstructure. We consider the same thermal gradient $(G=1 \mathrm{~K} / \mathrm{mm})$ and pulling velocity $\left(V_{p}=10 \mu \mathrm{m} / \mathrm{s}\right)$ than before. A simulation is performed as follows: first, the columnar front is stabilized at its steadystate undercooling. Then, inoculation on randomly distributed particles is activated. The particles have a concentration $c_{\text {inoc }}=1.25 \mathrm{~mm}^{-2}$ and a nucleation undercooling $\Delta T_{n}=0 \mathrm{~K}$. Unlike the simulations shown in Fig. 7, the grains first follow the circular germ regime before being replaced with a four-branches grain. During the CET depicted in Fig 9.a, the growth velocity and length of the red grains primary branches are represented in Fig. 9.b and Fig. 9.c. During the first stage of circular growth, the radial growth velocity of the grains is represented with continuous black lines and can be compared to the Ivantsov solution drawn with dashed black line. During short simulation times, the transient radial velocity is larger than the one predicted by the Ivantsov solution but after a short time $(\sim 150 \mathrm{sec})$, it becomes smaller than the Ivantsov prediction. At $t \sim 350 \mathrm{sec}$, the circular grains reach a critical size and are replaced by a randomly oriented dendritic grain formed of four orthogonal branches. The growth velocity of the grains primary branches is plotted with different colors depending of their orientations with respect to the thermal gradient. Just after the transition from circular to dendritic grains, the velocity jumps significantly due to the change of growth law associated to the different geometries (circular and dendritic). We note that the fact that the velocity at $t \sim 350 \mathrm{sec}$ is close to the one predicted by the Ivantsov solution is simply a coincidence influenced by the choice of the transition radius.
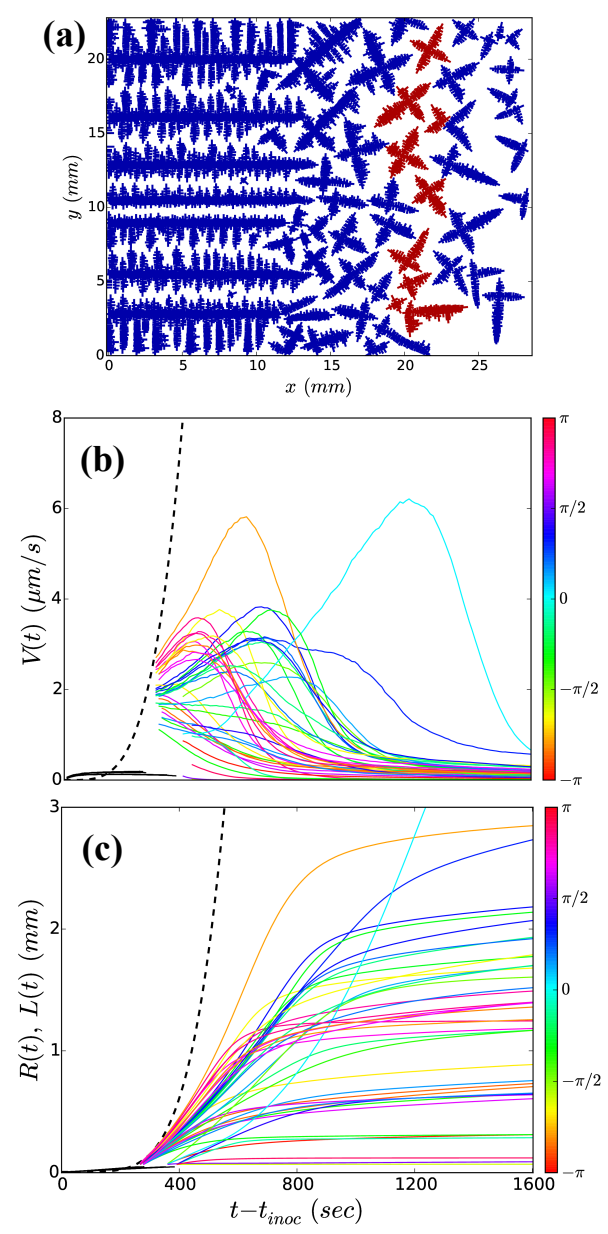

Figure 9: (a) Snapshot of the microstructure at $t \simeq 1600 \mathrm{sec}$ where the grains represented in red are the one considered in panels (b) and (c). Velocity (b) and length (c) of the germs (continuous black line) and the primary branches of developed equiaxed grains shown in red in panel (a). The different colors correspond to different orientations of the branches with respect to the thermal gradient direction as shown in the color bars of the plot. The black dash-lines represent the analytical prediction assuming that the growth follows the Ivantsov relation.

For most of the grains, the velocity of their branches increases progressively and do not follow the steep increase of the Ivantsov prediction. As explained previously, this is attributed to the history-dependent composition profile around the grains accounted for in our model 
and neglected in the Ivantsov solution. Due to the solutal interactions between the different grains, the growth velocity reaches a peak value and then decreases until the growth is stopped. For this choice of parameters, the velocity of the branches (and their resulting length) depend weakly on their orientation and is mainly influenced by the proximity of the neighboring grains as depicted also in Fig. 9.c. We note that this statement becomes untrue for different simulation parameters (in particular a larger thermal gradient) for which the equiaxed structure shows elongated grains in the direction of the thermal gradient (see for example Fig. 12).

When the growth of the primary branches is slowed down by the proximity of neighboring grains, their velocity and length is significantly lower than the Ivantsov prediction. The size of the equiaxed grains and therefore their ability to block the columnar front is over-estimated in the analytical models relying on the Ivantsov relation. However, this outcome depends on the parameters considered here (inoculant concentration, thermal gradient and pulling velocity). As an example, for much higher inoculant concentration, the growth of equiaxed grains could be stopped much earlier, where the Ivantsov solution underestimates their growth velocity. In this case, the Ivantsov solution and the analytical models relying on it underestimate the equiaxed grains size and therefore their ability to block the columnar front.

\section{Columnar-to-Equiaxed transition}

The DNN model can be used to investigate the CET and compare directly the resulting morphology with the outcome of analytical models. We consider that nucleation occurs on foreign inoculant particles randomly dispersed in the melt. We first consider an inoculant concentration $c_{\text {inoc }}=1.25 \mathrm{~mm}^{-2}$ with a nucleation undercooling $\Delta T_{n}=8 \mathrm{~K}$. To be consistent with the rest of the study, we transpose in 2D the analytical model of Gäumann, Trivedi and Kurz [5] (referred to as GTK model in the following). The different steps of this analytical approach are detailed in Appendix C. In this type of model, the transition is assumed to occur when the equiaxed grains cover a fraction between $\eta=1 \%$ and $\eta=99 \%$ of the columnar front. The corresponding transition lines are respectively represented as black dashed and solid lines in the microstructure diagram of Fig. 10.

DNN simulations are performed as follows: first, the columnar front is stabilized in the temperature gradient. Once a stationary structure is obtained, the nucleation of new grains on inoculant particles is activated, leading (or not) to the CET. The results of our DNN simulations for

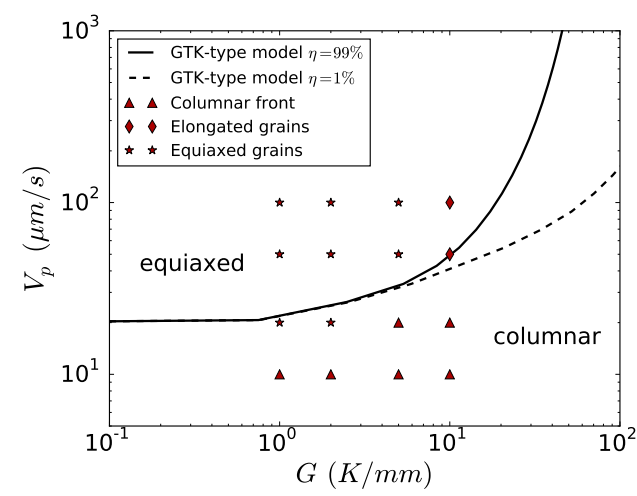

Figure 10: Comparison of the simulation output with the prediction of the 2D GTK model (see Appendix $\mathrm{C}$ for details) with inoculants of concentration $c_{\text {inoc }}=1.25 \mathrm{~mm}^{-2}$ and of nucleation undercooling $\Delta T_{n}=8 \mathrm{~K}$

different values of $V_{p}$ and $G$, reported with red symbols in Fig. 10, compare reasonably well with the prediction of the GTK model.

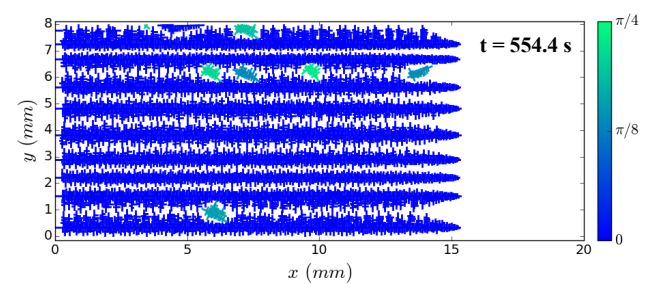

Figure 11: Microstructure obtained for $G=10 \mathrm{~K} / \mathrm{mm}, V_{p}=20 \mu \mathrm{m} / \mathrm{s}$, with an inoculant concentration $c_{\text {inoc }}=1.25 \mathrm{~mm}^{-2}$ and a nucleation undercooling $\Delta T_{n}=8 \mathrm{~K}$. The snapshot is taken at $t=554.4 \mathrm{~s}$ after the activation of nucleation.

In this range of parameters, the CET is mainly controlled by the relative value of the columnar front undercooling $\Delta T_{c}$ and the nucleation undercooling $\Delta T_{n}$ : if $\Delta T_{c}<\Delta T_{n}$, nucleation occurs behind the columnar front and the CET can not occur. The resulting microstructure is then columnar, possibly with some small misoriented grains nucleating in the inter-dendritic zone, as presented in Fig. 11.

For higher velocities, $\Delta T_{c}$ increases as shown in Fig. 6.b. For $\Delta T_{c} \gtrsim \Delta T_{n}$, nucleation occurs just in front of the columnar front. In this case, the equiaxed grains do not have the time to develop significantly before reaching the columnar front. As depicted in Fig. 12 (obtained for $G=10 \mathrm{~K} / \mathrm{mm}$ and $V_{p}=50 \mu \mathrm{m} / \mathrm{s}$ ), the columnar dendrites are progressively blocked by small grains that have nucleated in front of them. This leads to a progressive transition that spans over $7 \mathrm{~mm}$ as shown in the last panel 


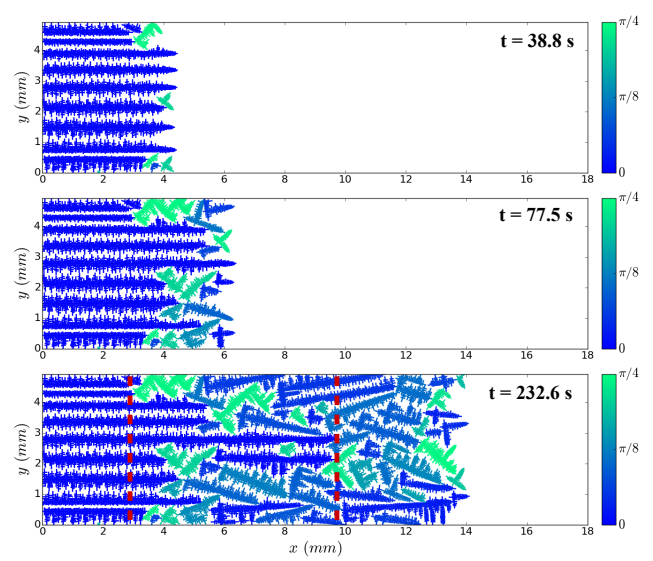

Figure 12: Microstructure obtained for $G=10 \mathrm{~K} / \mathrm{mm}, V_{p}=50 \mu \mathrm{m} / \mathrm{s}$, with an inoculant concentration $c_{\text {inoc }}=1.25 \mathrm{~mm}^{-2}$ and a nucleation undercooling $\Delta T_{n}=8 \mathrm{~K}$. The snapshots are taken at different times after the activation of nucleation, indicated in the upper right corners of the pictures. The progressive character of the CET is shown by two vertical dashed lines presenting the beginning and the end of the CET.

of Fig. 12. The new grains present an elongated morphology characterized by an average aspect ratio ${ }^{4}$ greater than 2. As such, this morphology is reported as "elongated grains" in Fig. 10. We also note that, for this set of parameters, a fraction of the inoculant particles do not lead to the formation of new grains. Because of the solutal interactions with neighboring grains, the condition of Eq. (28) is never fulfilled for some inoculant particles that remain inactivated.

For lower thermal gradients, we observe a much sharper transition as depicted in Fig. 13 (obtained for $G=2 \mathrm{~K} / \mathrm{mm}$ and $V_{p}=50 \mathrm{~mm} / \mathrm{s}$ ). The lower gradient allows a longer time for the equiaxed grains to grow before encountering the columnar front. The resulting CET is much sharper than presented in Fig. 12.

The discrepancy between the numerical results and the GTK model shown in Fig. 10 can be attributed to the underestimation of the columnar front undercooling by the Ivantsov solution as explained in section 4. This underestimation leads to a shift of the CET towards the equiaxed structures.

We then consider the limiting case of a low undercooling $\Delta T_{n}=0.196 \mathrm{~K}$, corresponding to an ideal athermal nucleation on a inoculant particle of radius $2 \mu \mathrm{m}$. In this situation, for any choice of $G$ and $V_{p}$, nucleation occurs

\footnotetext{
${ }^{4}$ We define the aspect ratio of a grain as the ratio between its length in the direction of the thermal gradient over its length in the perpendicular direction
}

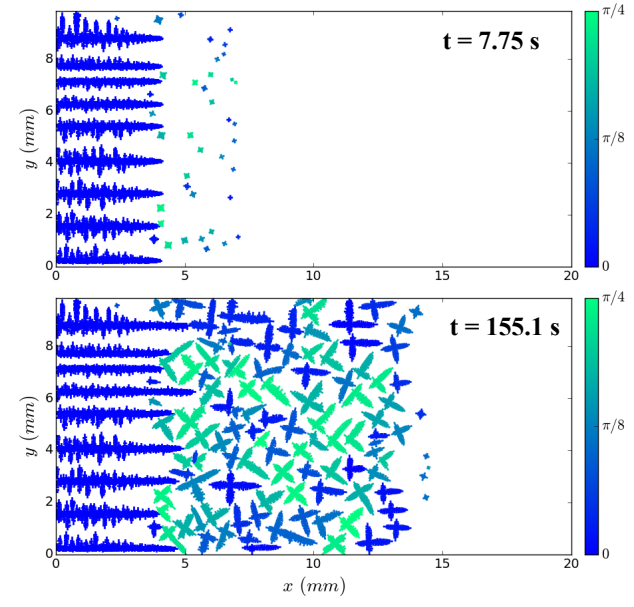

Figure 13: Microstructure for $G=2 \mathrm{~K} / \mathrm{mm}, V_{p}=50 \mu \mathrm{m} / \mathrm{s}$, an inoculant concentration $c_{\text {inoc }}=1.25 \mathrm{~mm}^{-2}$ of particles of nucleation undercooling $\Delta T_{n}=8 \mathrm{~K}$ for different times after the activation of nucleation.

in front of the columnar grains. With the previous inoculant concentration, the GTK model predicts an equiaxed morphology for the entire range of parameters explored in this study. Hence, we decrease the inoculant concentration to $0.125 \mathrm{~mm}^{-2}$ to investigate the CET in the limit of low inoculant concentration and low nucleation undercooling. Figure 14 shows the microstructure diagram obtained from our simulations compared to the analytical solution from the GTK model. As mentioned previously, the low gradient and high velocities favor an equiaxed structure while high gradient and low velocities promote the nucleation of elongated grains presenting an aspect ratio greater than two. In this regime of low inoculant concentration and small undercooling, the CET is controlled by the growth of the equiaxed grains in front of the columnar front. We note that even at high gradient and low velocity, the columnar front is progressively blocked by misoriented grains as shown in Fig. 15. In this regime, growth competition between grains becomes important. In the first panel of Fig. 15, the grain circled in black has been overgrown by the neighboring columnar dendrites. However, the two grains circled in red have not been overgrown, despite an undercooling larger than the well oriented grains. By a tertiary branching mechanisms, these grains "survive" and adopt an elongated morphology, then promoting the development of elongated grains in front of the columnar structure. This observation can be related to recent phase-field studies of grain competition in 2D [32] and 3D [33]. The authors show that the overgrowth of misoriented grains by well-oriented grains is not as simple as considered in analytical models [1] and 


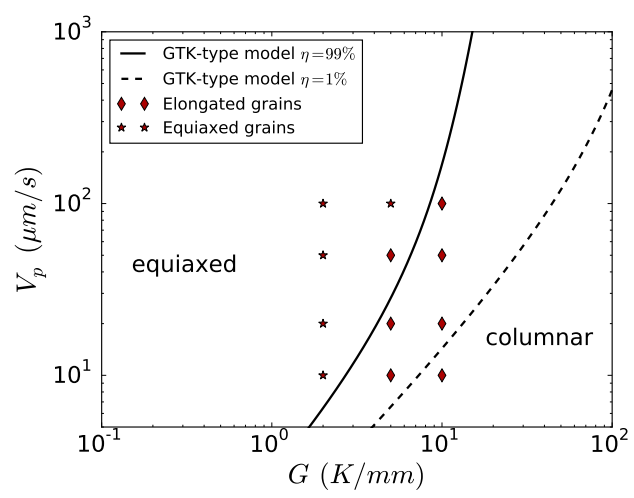

Figure 14: Comparison of the simulation results with the prediction of the 2D GTK model (see Appendix C for details) with inoculant concentration $c_{\text {inoc }}=0.125 \mathrm{~mm}^{-2}$ and nucleation undercooling $\Delta T_{n}=$ $0.196 \mathrm{~K}$.

has to be thought as a stochastic process controlled by tertiary branching of the grains.

\section{Summary and conclusion}

In the first part of this paper, we have used the DNN model $[16,17]$ to investigate the CET in $2 \mathrm{D}$ and discuss the assumptions used in analytical models and most of the coarse-grained numerical approaches. This meso-scale technique allows to reach the relevant length and time scales while relying on a minimal number of assumptions. In particular, it accounts for the complex dendritic grain structure, the transient growth regime of the dendrites and the solutal interactions between the grains. In addition, the DNN approach has been extended to account for the nucleation of new grains and the growth of misoriented dendrites.

We first investigated the primary spacing and undercooling of the columnar front for different pulling velocities and thermal gradients. We showed that the interactions with neighboring dendrites and higher-order branches lead to an increase of the columnar front undercooling. We then investigated the growth of equiaxed grains in a thermal gradient and showed that it does not follow the analytical Ivantsov relation as assumed in most of the analytical and coarse-grained numerical approaches of CET. Finally, we have used our DNN model to study the CET for two different inoculant populations. In the case of high inoculant concentration and large nucleation undercooling, the CET was found to be mainly attributed to the difference between the columnar front and nucleation undercoolings: when nucleation occurs behind the columnar front, no CET can occur. Also, simulations

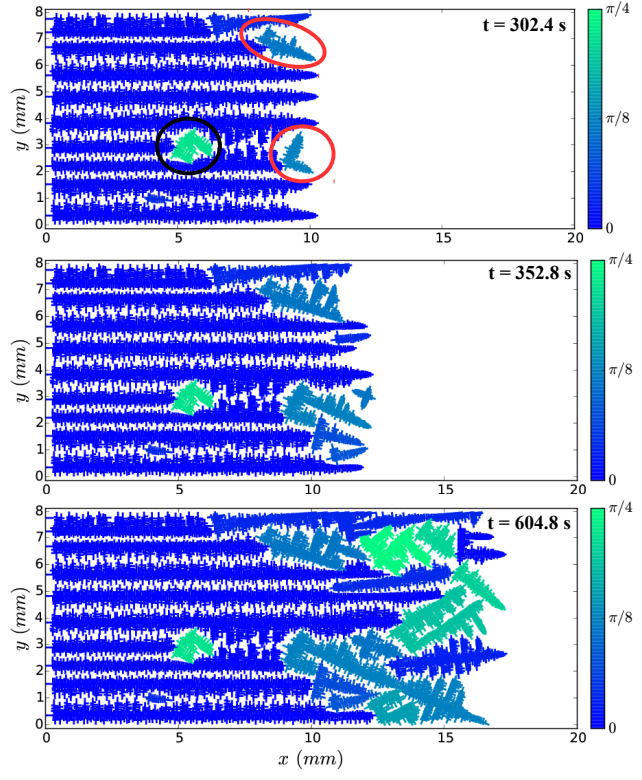

Figure 15: Microstructure obtained for $G=10 \mathrm{~K} / \mathrm{mm}, V_{p}=20 \mu \mathrm{m} / \mathrm{s}$, an inoculant concentration $c_{\text {inoc }}=0.125 \mathrm{~mm}^{-2}$ of nucleation undercooling $\Delta T_{n}=0.196 \mathrm{~K}$ for different times after nucleation is activated.

yielded various types of CET (progressive and sharp), depending on how far nucleation occurs compared to the position of the columnar front in the thermal gradient. In the case of a low inoculant concentration and low undercooling, nucleation always occurs in front of the columnar grains and the CET is then mainly controlled by grain growth competition. Indeed, as shown in previous phase-field studies [32,33], a misoriented grain can be slowly overgrown by well-oriented grains because of tertiary branching mechanisms. The nucleation of new grains in front of the columnar front then leads to a progressive CET as shown in Fig. 15. This study demonstrates that some of the assumptions widely used in analytical and most numerical approaches are questionable.

However, this work has been performed in two dimensions and the scaling laws for transient and steady-state dendritic growth are different in 3D. First, the primary spacings of the columnar structure is larger in $2 \mathrm{D}$ than in $3 \mathrm{D}$ due to the longer range solutal interactions between dendrites [34]. For the same reason, the influence of neighboring dendrites and side branches on the columnar front undercooling is expected to be different in $2 \mathrm{D}$ and 3D. The duration and the influence of the transient growth regime are also known to be amplified in 2D. The mechanisms of grain competition observed for low inoculant concentration in section 6 are also dimension-dependent: in $3 \mathrm{D}$, the presence of a second misorientation angle can 
indeed favor the overgrowth of misoriented grains by the columnar grains. The DNN model has been extended to 3D [17] and its application to investigate the CET is presented in part II of this paper [35], where we investigate the magnitude of the aforementioned effects in 3D and compare quantitatively the model results to experimental observations.

\section{Acknowledgment}

This research was supported by NASA under award No. 80NSSC19K0135 in the framework of the CETSOL project. The authors would like to thank Damien Tourret for his help and remarks during the development of the model and comments on the manuscript.

\section{Appendix A. Integration contours for different needle orientations}

In practice, the distances $a_{1}$ and $a_{2}$ have to be computed for every needle and depend on the relative position of the needle and the integration contour. Numerically, we distinguish two different cases depending on the magnitude of the angle $\theta$ between the direction of the needle and one of the main directions of the finite difference grid.

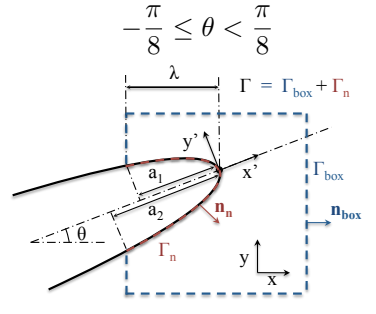

(a)

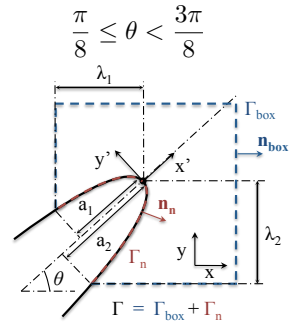

(b)
Figure A.16: Shape of the integration box chosen to compute numerically the flux intensity factor depending on the angle $\theta$ between the needle and one of the main direction of the square finite difference grid.

For the values of $\theta=-\pi / 8 \leq \theta<\pi / 8$, the integration box is chosen such that the intersection between the box and the needle only occurs on one side of the box as depicted in Fig. A.16.a. We introduce the distance $\lambda$ between the tip of the needle and the side of the box where the needle intersects the integration box. The values of $a_{1}$ and $a_{2}$ are obtained by equating the equation of the parabola in the frame $\left(x^{\prime}, y^{\prime}\right)$ and the straight line representing the left side of the integration contour:

$$
\pm \sqrt{-2 \rho x^{\prime}}=\frac{x^{\prime}}{\tan \theta}+\frac{\lambda}{\sin \theta}
$$

This can be rewritten as a second degree equation on $x^{\prime}$ which can be easily solved to obtain $a_{1}$ and $a_{2}$ :

$$
\begin{aligned}
& a_{1}=\frac{\rho \sin ^{2} \theta+\lambda \cos \theta-\sin \theta \sqrt{\rho^{2} \sin ^{2} \theta+2 \rho \lambda \cos \theta}}{\cos ^{2} \theta} \\
& a_{2}=\frac{\rho \sin ^{2} \theta+\lambda \cos \theta+\sin \theta \sqrt{\rho^{2} \sin ^{2} \theta+2 \rho \lambda \cos \theta}}{\cos ^{2} \theta}
\end{aligned}
$$

For values of $\theta$ such that $\pi / 8 \leq \theta<3 \pi / 8$, the integration box is sized such that the intersection between the contour and the needle occurs on two adjacent sides as depicted in Fig. A.16.b. We then introduce the distances $\lambda_{1}$ and $\lambda_{2}$ between the tip of the needle and the two sides of the integration box. Equating the expression of the parabola and the straight lines yields:

$$
\begin{aligned}
& a_{1}=\frac{\rho \sin ^{2} \theta+\lambda_{1} \cos \theta-\sin \theta \sqrt{\rho^{2} \sin ^{2} \theta+2 \rho \lambda_{1} \cos \theta}}{\cos ^{2} \theta} \\
& a_{2}=\frac{\rho \cos ^{2} \theta+\lambda_{2} \cos \theta-\cos \theta \sqrt{\rho^{2} \cos ^{2} \theta+2 \rho \lambda_{2} \sin \theta}}{\sin ^{2} \theta}
\end{aligned}
$$

\section{Appendix B. Sensitivity to the destabilization radius}

During the first stage of growth of equiaxed grains, the grain is considered to keep a circular shape up to a point where its radius reach a critical value set at $R_{c}=8.7 \rho_{s}$ where $\rho_{s}$ is the stationary state tip radius. It is then replaced by a cross-shaped grain of four orthogonal needles of tip radius $\rho_{s}$. The length of the dendritic arms is chosen such that the solid fraction of the grain is the same between its circular and needle representations. Figure B.17 shows the evolution of a grain in isothermal conditions considering three different values of $R_{c}=5,10,15 \rho_{s}$. The resulting morphology is practically identical in the three cases, showing the small sensitivity of the grain evolution on $R_{c}$.

\section{Appendix C. 2D GTK model}

This appendix presents the equations of the GTK model [5] transposed in 2D. This model relies on several steps that are details in the following: 


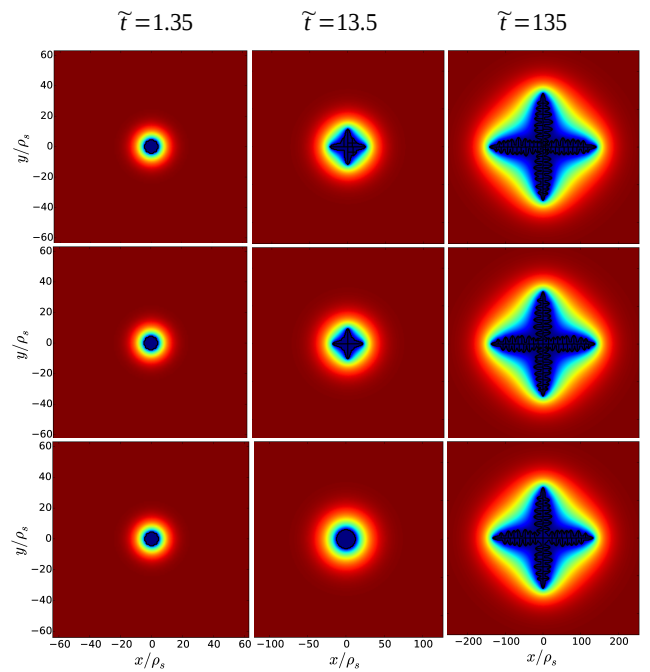

Figure B.17: Grain evolution for different destabilization radius: $R=$ $5 \rho_{s}$ (top row), $R=10 \rho_{s}$ (middle row) and $R=15 \rho_{s}$ (bottom row) at times $\tilde{t}=1.35$ (left column), $\tilde{t}=13.5$ (middle column) and $\tilde{t}=135$ (right column).

\section{Appendix C.1. Columnar front}

The primary dendrites of the columnar front are characterized by their tip radius $\rho$, their tip supersaturation $\Omega$ and the concentration of the liquid at the dendritic tip $u_{t}$. Assuming that the primary dendrites of the columnar front do not interact with each others and with the rest of the dendritic microstructure, their growth follows the Ivantsov relation in $2 \mathrm{D}$. Together with the micro-solvability condition and the definition of the tip supersaturation, the steady-state values of $\rho_{s}, \Omega_{s}$ and $u_{s}$ are related through the following set of equations:

$$
\begin{aligned}
\rho_{s} & =\sqrt{\frac{1}{1-(1-k) u_{s}} \frac{2 D d_{0}}{\sigma V_{p}}} \\
\Omega_{s} & =I v\left(\frac{\rho_{s} V_{p}}{2 D}\right) \\
u_{s} & =\frac{1-\Omega_{s}}{1-(1-k) \Omega_{s}}
\end{aligned}
$$

where $I v$ denote the Ivantsov function in 2D: $I v(P e)=$ $\sqrt{\pi P e} \exp (P e) \operatorname{erfc}(\sqrt{P e})$ and the meaning of the other parameters variables is detailed in section 2 .

The numerical solution of this set of equations with an iterative method yields the steady-state tip concentration $u_{s}$. We can then deduce the position of the columnar front in the frame of the sample, $x_{c}(t)=u_{s} l_{T}+V_{p} t$ (where $l_{T}=|m|(1-k) c_{l}^{0} / G$ denotes the thermal length), and in the frame associated to the isotherms: $x_{c}^{\prime}=u_{s} l_{T}$ (see Fig. 1).

\section{Appendix C.2. Nucleation}

The innovative aspect of the GTK model compared to the first approaches of Hunt and Flood [1, 2, 3, 4] consists in accounting for the influence of the diffusion profile in front of the columnar grains on the nucleation and the growth of the equiaxed grains. The steady-state Ivantsov solution [1] gives the following expression for the concentration profile ahead of the columnar front:

$$
u\left(x^{\prime}\right)=1+\left(u_{s}-1\right) \frac{\operatorname{erfc}\left(\sqrt{V_{p}\left(x^{\prime}-u_{s} l_{T}\right) / D}\right)}{\operatorname{erfc}(\sqrt{2 P e})}
$$

We assume that the nucleation of new grains occurs on inoculant particles activated at a fixed undercooling denoted $\Delta T_{n}$. Following the same steps as in section 3.1, nucleation occurs on a particle located in $x^{\prime}$ if the concentration satisfies

$$
u\left(x^{\prime}\right)>\frac{x^{\prime}}{l_{T}}+\frac{\Delta T_{n}}{|m|(1-k) c_{l}^{0}}
$$

\section{Appendix C.3. Growth of equiaxed grains}

The size of the equiaxed grains is assumed to be small compared to the thermal length such that the different branches of the grain grow in the same temperature environment. Moreover, it is assumed that the grains grow without interacting with each other and that the temperature environment around them evolves slowly such that their growth is dictated by the steady-state Ivantsov solution in isothermal conditions with a solute concentration given by C.4. The $x$-dependent supersaturation felt by the equiaxed grains is then given by

$$
\Omega_{e g}\left(x^{\prime}\right)=\frac{c_{l}\left(T\left(x^{\prime}\right)\right)-c_{l}^{0}+u\left(x^{\prime}\right)(1-k) c_{l}^{0}}{(1-k) c_{l}\left(T\left(x^{\prime}\right)\right)} .
$$

The growth of the branches of the equiaxed grains is then determined from the combination of the 2D Ivantsov relation and the micro-solvability condition:

$$
\begin{gathered}
\Omega_{e g}\left(x^{\prime}\right)=I v\left(\frac{\rho V}{2 D}\right) \\
\rho^{2} V=\frac{2 D d_{0}}{\sigma},
\end{gathered}
$$

yielding

$$
V\left(x^{\prime}\right)=\frac{2 D \sigma}{d_{0}}\left(I v^{-1}\left(\Omega_{e g}\left(x^{\prime}\right)\right)\right)^{2} .
$$

We note that for small Péclet numbers, the Ivantsov relation can be approximated by $I v(P e) \simeq \sqrt{\pi P e}$, therefore $V\left(x^{\prime}\right) \sim \Omega_{e g}\left(x^{\prime}\right)^{4}$, explaining the sharp increase of the velocity depicted in Fig. 7.a. 
Appendix C.4. Blocking of the columnar front by the equiaxed grains

The CET is then assessed by considering the size of the equiaxed grains when they meet the columnar front at the position $x_{c}^{\prime}$, given in Appendix C.1. Assuming that the initial radius of the grains is negligibly small, the radius of the grains is obtained by integrating their growth rate between $x_{n}^{\prime}$ and $x_{c}^{\prime}$ :

$$
R_{e g}\left(x_{c}^{\prime}\right)=\frac{1}{V_{p}} \int_{x_{n}^{\prime}}^{x_{c}^{\prime}} V\left(x^{\prime}\right) d x^{\prime} .
$$

When they meet the columnar front, the equiaxed grains cover approximately a fraction of the space given by

$$
\eta\left(x_{c}^{\prime}\right)=c_{\text {inoc }} \pi R_{e g}^{2}\left(x_{c}^{\prime}\right)
$$

where $c_{\text {inoc }}$ is the number of inoculant particles per surface area. The CET is then assumed to occur if $\eta_{e g}$ is greater than a given threshold, generally considered between $1 \%$ and $99 \%$. The plot in Fig. 10 and 14 are obtained by fixing a constant velocity $V_{p}$ and finding numerically the thermal gradient $G$ satisfying the relation $\eta_{e g}\left(x_{c}^{\prime}\right)=X$ with $X=$ $1 \%$ or $X=99 \%$ (we note that the thermal gradient enters the equations at several places in a complex manner since it enters in the definition of the thermal length $l_{T}=|m|(1-$ k) $\left.c_{l}^{0} / G\right)$.

\section{References}

[1] J. Dantzig, M. Rappaz, Solidification, Engineering Sciences, EPFL press, 2009.

URL http://www. solidification.org/

[2] J. Hunt, Steady state columnar and equiaxed growth of dendrites and eutectic, Mater. Sci. Eng. 65 (1984) 75-83. doi:10.1016/0025-5416(84)90201-5.

[3] S. Flood, J. Hunt, Columnar and equiaxed growth: II. Equiaxed growth ahead of a columnar front, J. Cryst. Growth 82 (1987) 552-560. doi:10.1016/00220248(87)90347-2.

[4] S. Flood, J. Hunt, Columnar and equiaxed growth: I. A model of a columnar front with a temperature dependent velocity, J. Cryst. Growth 82 (1987) 543. doi:10.1016/0022-0248(87)90346-0.

[5] M. Gäumann, R. Trivedi, W. Kurz, Nucleation ahead of the advancing interface in directional solidification, Mater. Sci. Eng. A A226-228 (1997) 763-769. doi:10.1016/S0921-5093(97)80081-0.

[6] C. Wang, C. Beckermann, A multiphase solute diffusion model for dendritic alloy solidification, Metall. Trans. A 24A (1993) 2787-2802. doi:10.1007/BF02659502.
[7] C. Wang, C. Beckermann, Prediction of columnar to equiaxed transition during diffusion-controlled denditric alloy solidification, Metall. Mater. Trans. A 25 (1994) 1081-1093. doi:10.1007/BF02652282.

[8] M. Martorano, C. Beckermann, C. Gandin, A solutal interaction mechanism for the columnar-to-equiaxed transition in alloy solidification, Metall. Mater. Trans. A 34 (2003) 1657-1674. doi:10.1007/s11661-003-0311-x.

[9] C. Gandin, M. Rappaz, A coupled finite elementcellular automaton model for the prediction of dendritic grain structures in solidification processes, Acta Metall. Mater. 42 (7) (1994) 2233-2246. doi:10.1016/09567151(94)90302-6.

[10] M. Vandyoussefi, A. Greer, Application of cellular automaton-finite element model to the grain refinement of directionally solidified Al4.15 wt\% Mg alloys, Acta Mater. 50 (2002) 1693-1705. doi:10.1016/S13596454(02)00015-0.

[11] H. Dong, P. Lee, Simulation of the columnarto-equiaxed transition in directionally solidified $\mathrm{AlCu}$ alloys, Acta Mater. 53 (3) (2005) 659-668. doi:10.1016/j.actamat.2004.10.019.

[12] A. Badillo, C. Beckermann, Phase-field simulation of the columnar-to-equiaxed transition in alloy solidification, Acta Mater. 54 (8) (2006) 2015-2026. doi:10.1016/j.actamat.2005.12.025.

[13] J. Li, J. Wang, G. Yang, Phase-field simulation of microstructure development involving nucleation and crystallographic orientations in alloy solidification, J. Cryst. Growth 309 (1) (2007) 65-69. doi:10.1016/j.jcrysgro.2007.08.025.

[14] W. Boettinger, J. Warren, C. Beckermann, A. Karma, Phase-field simulation of solidification, Ann. Rev. Mater. Res. 32 (1) (2002) 163-194. doi:10.1146/annurev.matsci.32.101901.155803.

[15] A. Karma, D. Tourret, Atomistic to continuum modeling of solidification microstructures, Curr. Opin. Solid. St. M. 20 (1) (2015) 25-36. doi:10.1016/j.cossms.2015.09.001.

[16] D. Tourret, A. Karma, Multiscale dendritic needle network model of alloy solidification, Acta Mater. 61 (17) (2013) 6474-6491. doi:10.1016/j.actamat.2013.07.026.

[17] D. Tourret, A. Karma, Three-dimensional dendritic needle network model for alloy solidification, Acta Mater. 120 (2016) 240-254. doi:10.1016/j.actamat.2016.08.041.

[18] M. Gündüz, J. Hunt, The measurement of solid-liquid surface energies in the al-cu, al-si and pb-sn systems, Acta Metall. 33 (9) (1985) 1651-1672. doi:10.1016/00016160(85)90161-0. 
[19] C.-A. Gandin, From constrained to unconstrained growth during directional solidification, Acta Mater. 48 (10) (2000) 2483-2501. doi:10.1016/S1359-6454(00)00070-7.

[20] G. Zimmermann, L. Sturz, B. Billia, N. Mangelinck-Noel, H. Nguyen Thi, C.-A. Gandin, D. Browne, W. Mirihanage, Investigation of columnar-to-equiaxed transition in solidification processing of AlSi alloys in microgravitythe CETSOL project, in: Journal of Physics: Conference Series, Vol. 327, 2011, p. 012003. doi:10.1088/1742$6596 / 327 / 1 / 012003$.

[21] G. Zimmermann, L. Sturz, B. Billia, N. MangelinckNoel, D. Liu, H. Nguyen Thi, N. Bergeon, C.-A. Gandin, D. Browne, C. Beckermann, et al., Columnar-to-equiaxed transition in solidification processing of AlSi7 alloys in microgravity the CETSOL project, in: Mater. Sci. Forum, Vol. 790, Trans Tech Publ, 2014, pp. 12-21. doi:10.4028/www.scientific.net/MSF.790-791.12.

[22] D. R. Liu, N. Mangelinck-Noël, C.-A. Gandin, G. Zimmermann, L. Sturz, H. Nguyen Thi, B. Billia, Structures in directionally solidified Al-7wt.\% Si alloys: Benchmark experiments under microgravity, Acta Mater. 64 (2014) 253 265. doi:10.1016/j.actamat.2013.10.038.

[23] G. Zimmermann, L. Sturz, H. Nguyen-Thi, N. Mangelinck-Noel, Y. Li, C.-A. Gandin, R. Fleurisson, G. Guillemot, S. Mcfadden, R. Mooney, et al., Columnar and Equiaxed Solidification of Al-7 wt.\% Si Alloys in Reduced Gravity in the Framework of the CETSOL Project, JOM 69 (8) (2017) 1269-1279. doi:10.1007/s11837-017-2397-4.

[24] A. Barbieri, J. Langer, Predictions of dendritic growth rates in the linearized solvability theory, Phys. Rev. A 39 (10) (1989) 5314. doi:10.1103/PhysRevA.39.5314.

[25] M. Plapp, A. Karma, Multiscale random-walk algorithm for simulating interfacial pattern formation., Phys. Rev. Lett. 84 (8) (2000) 1740-1743. doi:10.1103/PhysRevLett.84.1740.

[26] Y. Couder, J. Maurer, R. González-Cinca, A. HernándezMachado, Side-branch growth in two-dimensional dendrites. I. Experiments, Phys. Rev. E 71 (3) (2005) 031602. doi:http://dx.doi.org/10.1103/PhysRevE.71.031602.

[27] A. Greer, A. Bunn, A. Tronche, P. Evans, D. Bristow, Modelling of inoculation of metallic melts: application to grain refinement of aluminium by $\mathrm{AlTiB}$, Acta Mater. 48 (11) (2000) 2823-2835. doi:10.1016/S1359-6454(00)00094-X.

[28] A. Greer, P. Cooper, M. Meredith, W. Schneider, P. Schumacher, J. Spittle, A. Tronche, Grain refinement of aluminium alloys by inoculation, Adv. Eng. Mat. 5 (1-2) (2003) 81-91. doi:10.1002/adem.200390013.
[29] B. McKay, P. Cizek, P. Schumacher, K. O'Reilly, Heterogeneous nucleation in an al-ni-si alloy studied using a metallic glass technique, Mat. Sci. Eng. A 304 (2001) 240-244. doi:10.1016/S0921-5093(00)01476-3.

[30] H. Jung, N. Mangelinck-Noël, C. Bergman, B. Billia, Determination of the average nucleation undercooling of primary al-phase on refining particles from al-5.0 wt\% ti-1.0 wt $\%$ b in al-based alloys using dsc, J. Alloys Compd. 477 (1) (2009) 622-627. doi:10.1016/j.jallcom.2008.10.109.

[31] C. Zener, Theory of Growth of Spherical Precipitates from Solid Solution, J. Appl. Phys. 20 (10) (1949) 950. doi:10.1063/1.1698258.

[32] D. Tourret, A. Karma, Growth competition of columnar dendritic grains: A phase-field study, Acta Mater. 82 (2015) 64-83. doi:10.1016/j.actamat.2014.08.049.

[33] D. Tourret, Y. Song, A. Clarke, A. Karma, Grain growth competition during thin-sample directional solidification of dendritic microstructures: A phasefield study, Acta Mater. 122 (2017) 220-235. doi:10.1016/j.actamat.2016.09.055.

[34] D. Tourret, A. Karma, A. Clarke, P. Gibbs, S. Imhoff, Three-dimensional dendritic needle network model with application to al-cu directional solidification experiments, IOP Conf. Ser. Mat. Sci. Eng. 84 (2015) 012082. doi:10.1088/1757-899X/84/1/012082.

[35] C.-H. Chen, A. Molavi Tabrizi, P.-A. Geslin, A. Karma, Dendritic Needle Network Modeling of the Columnar-toEquiaxed Transition. Part II: Three Dimensional Formulation, Implementation and Comparison With Experiments, Acta Mater. 